\title{
An Inventory Model for Deteriorating Items with Stock and Price Sensitive Demand
}

\author{
R. Roy Chowdhury • S. K. Ghosh • K. S. Chaudhuri
}

Published online: 31 October 2014

(C) Springer India Pvt. Ltd. 2014

\begin{abstract}
It is commonly seen that racks overflowing with large quantity of commodities, displayed in the market attracts more customers. This behavior of the customer suggests that demand varies in proportion with stock level. Also the inventory is subjected to deterioration which leads to some amount of loss to the firm. Taking all these points under consideration, a firm should seriously think of its pricing and ordering strategy, as the demand is highly influenced by the selling price and the inventory level of the commodity. This paper aims to develop an inventory model for finding the strategy for a firm that sells a seasonal item over a finite planning time. Keeping in mind the deterioration rate,the purpose of the paper is to develop a model which maximizes the firm's expected profit by determining the optimal ordering quantity and price setting/changing strategy.
\end{abstract}

Keywords Displayed stock · Price setting · Deterioration · Inventory level

\section{Introduction}

Display of items on the racks in markets have a persuasive effect on a customer who tends to buy more. In other words, seeing large quantity of items on display a customer gets motivated to purchase in bulks. According to Levin et al. [8] "At times, the presence of inventory has a motivational effect on the people around it. It is a common belief that

\footnotetext{
R. R. Chowdhury

Department of Mathematics, Techno India College of Technology,

Newtown, Rajarhat, Kolkata 700156, India

e-mail: ruma_roych@yahoo.co.in

S. K. Ghosh $(\varangle)$

Department of Mathematics, The Bhawanipur Education Society College, Kolkata 700020, India

e-mail: santanu_96@yahoo.co.in
}

K. S. Chaudhuri

Department of Mathematics, Jadavpur University, Kolkata 700 032, India

e-mail: chaudhuriks@gmail.com 
large piles of goods displayed in a supermarket will lead the customer to buy more." Many inventory modelers have made several attempts to analyze the inventory models assuming a functional relation between the demand rate and the on-hand inventory (stock-level). These researchers were attracted by this observation and many inventory models have been developed in this direction. Silver and Peterson [23] noted that sales at the retail level tend to be proportional to inventory displayed. Stock-dependent demand patterns were considered by several other researches like, Gupta and Vrat [7], Baker and Urban [1], Mandal and Phaujder [12,13], Datta and Pal [4,5] and Urban [25], 1995a, b, [26,27], Pal et al. [14] etc. Roy and Chaudhuri [15] developed inventory model with stock-dependent demand, shortages, inflation and time-discounting. Roy and Chaudhuri [16] extended this model for the case of deteriorating items. Dye and Ouyang [6] developed inventory model for perishable items under stock-dependent selling rate and time-dependent partial backlogging. Sarkar $[18,19]$ developed a model with permissible delay in payment and stock dependent demand within the economic order quantity (EOQ) framework. But putting so much stock on display has its own shortcomings, such as loss due to deterioration and inventory holding cost.

Hence, speaking of the loss due to deterioration , an inventory model of two-warehouse with quadratically increasing demand and time varying deterioration were considered by Sett et al. [22]. Sarkar [21] also developed a production-inventory model with probabilistic deterioration in two-echelon supply chain management. Mishra [11] developed an inventory model which used preservation technology to reduce the deterioration rate of the instantaneous deteriorating items of the inventory.

Also, another vital factor that influences demand is selling price. As pricing is an obvious strategy to influence demand, studies on inventory models with price-dependent demand have received much attention. Sana [17] developed inventory model for salesmen's effort with stock and price sensitive demand. Dynamic pricing with real-time demand learning was developed by Lin [9].

Thus the market researchers have to investigate the factors that influence demand pattern, because customers purchasing behavior may be affected by factors such as selling price, inventory level, seasonality, and so on. Bhunia and Maiti [2] investigated a deteriorating inventory model with linear stock and time dependent demand. Mandal and Maiti [10] studied a production model with power form stock dependent demand. Chung et al. [3] considered a deteriorating inventory with linear stock dependent demand. Loss due to deterioration has been considered in this present model.

Since a firm may use a pricing strategy to spur demand for its seasonal goods, the inventory problems with price and stock dependent demand cannot be ignored. Urban and Baker [24] investigated a deterministic inventory problem in which the demand is a multivariate function of price, time and inventory level. Sarkar and Moon [20] developed an EPQ model with inflation in an imperfect production system

It is noted that the literature herein rarely considers the cases with multiple price changes, when the demand is price and stock dependent. Also changing price multiple number of times involve certain costs such as changing tags of the items,advertising the new costs to make the customers aware of the new lucrative price etc. All these factors has been taken into consideration while developing the model under the head-"price setting cost".

In the proposed model, the research highlights are as follows:

(1) Pricing strategy, stock dependent demand and loss due to deterioration have been brought together. 
(2) This paper studies a single replenishment inventory model in which a firm may reset its selling price to spur demand when consumers purchasing behavior are price and stock dependent.

(3) In this paper we develop three cases in which the selling price has been set and reset once, twice and thrice in a finite time zone.

(4) The purpose of this paper is to develop the solution procedure for determining the optimal order size and optimal selling prices for a seasonal item.

(5) The optimal solution is then illustrated with the help of numerical examples.

(6) The sensitivity analysis of the optimal solution towards the changes in the values of different system parameters is also studied.

\section{Assumptions and Notation}

The following assumptions and notation are used in the proposed model.

Assumptions

(1) A single replenishment inventory model is developed.

(2) Demand for the item is assumed to be price and stock dependent.

(3) The decision variables are the optimal order size and optimal selling prices to be set at certain intervals for a seasonal item.

(4) The inventory model is developed over a finite time horizon L.

(5) Shortages ate not permitted.

Notation

$Q: \quad$ Order quantity

$L: \quad$ The planning time interval in days

$n: \quad$ Total number of periods

$T: \quad$ Length of a period in days, $\mathrm{T}=\mathrm{L} / \mathrm{n}$

$j: \quad \quad \quad$ Period index, period $\mathrm{j}$ refers to the time interval $[(\mathrm{j}-1) \mathrm{T}, \mathrm{jT}]$

$W: \quad$ Selling price in $\$$ per unit per unit time

$p_{j}: \quad$ Selling price in $\$$ set during jth period

$\lambda_{j}(p, t)$ : Demand rate at time t of period $\mathrm{j}$ when the initial selling price is set at $\$ \mathrm{p}$

$h: \quad$ Unit inventory carrying cost (in \$) per unit time

$c: \quad$ Unit purchasing cost in $\$$

$k: \quad$ Pricing setting cost in $\$$

$\theta: \quad$ Deterioration rate

$h: \quad$ Holding cost (in \$) per unit item per unit time

$c: \quad$ Unit purchasing cost in $\$$

$K: \quad$ Incurred cost (in \$) associated with each price setting/adjustment

$I_{j}(t): \quad$ The inventory level at time $\mathrm{t}$ of period $\mathrm{j}$.

\section{Formulation and Solution of the Model}

In this section, we develop a mathematical model of the problem. Suppose a firm purchases $\mathrm{Q}$ units of a seasonal item and sells them over a finite time horizon L. Demand for the item 
is assumed to be price and stock dependent. The planning horizon $\mathrm{L}$ is divided into $n \leq \bar{n}$ equal time periods, each with $\mathrm{T}=\mathrm{L} / \mathrm{n}$ time units. The firm sets an initial selling price at the beginning of the period 1 . At the beginning of the subsequent periods, the firm resets its selling price. The selling price set during period $\mathrm{j}$ is denoted by $p_{j}$. We consider that the inventory is subjected to a constant deterioration rate $\theta$. In addition, assume that the firm sets the order quantity and the selling price at $Q$ and $\mathbf{p}=\left(p_{1}, p_{2}, \ldots, p_{n}\right)$, respectively. The demand rate at time $t$ of the period $\mathrm{j}$ is given by $\lambda_{j}\left(t, p_{j}\right)=\alpha-\beta p_{j}+\eta I_{j}(t), \quad 0 \leq t \leq T$. Let $q_{j}(t)$ be the inventory level at the start of period $\mathrm{j}$. Then, we have for the period $\mathrm{j}$, the amount of inventory is given by the following differential equation

$$
\frac{d I_{j}(t)}{d t}+\theta I_{j}(t)=-\left(\alpha-\beta p_{j}+\eta I_{j}(t)\right)
$$

If $q_{j}$ be inventory level at the start of period $\mathrm{j}$, then $I_{j}(0)=q_{j}$ and so the solution of equation (1) is given by

$$
I_{j}(t)=q_{j} \cdot e^{-(\theta+\eta) t}-\frac{\left(\alpha-\beta p_{j}\right)}{(\theta+\eta)}\left(1-e^{-(\theta+\eta) t}\right)
$$

Since $I_{(j-1)}(T)=I_{j}(0)=q_{j}$,

$$
I_{(j-1)}(T)=q_{(j-1)} e^{-(\theta+\eta) T}-\frac{\left(\alpha-\beta p_{j-1}\right)}{(\theta+\eta)}\left(1-e^{-(\theta+\eta) T}\right)
$$

Therefore

$$
q_{j}=q_{(j-1)} x^{-1}-\frac{\left(1-x^{-1}\right)\left(\alpha-\beta p_{j-1}\right)}{(\theta+\eta)}
$$

where $x=e^{(\theta+\eta) T}$

To reduce the unknown term $q_{j}$ in equation(3), we will reexpress $q_{j}$ in terms of $\mathrm{Q}$ and $\mathrm{p}$. Since initial inventory level is $\mathrm{Q}$, we have $q_{1}=Q$. Thus

$$
q_{2}=x^{-1} q_{1}-\frac{\left(1-x^{-1}\right)\left(\alpha-\beta p_{1}\right)}{(\theta+\eta)}=x^{-1} Q-\frac{\left(1-x^{-1}\right)\left(\alpha-\beta p_{1}\right)}{(\theta+\eta)}
$$

Let

$$
\hat{q_{j}}=x^{-(j-1)} Q-\frac{\alpha\left(1-x^{-(j-1)}\right)}{(\theta+\eta)}+\frac{\left(1-x^{-1}\right) \sum_{k=2}^{\mathrm{J}} x^{-(j-k)} \beta p_{k-1}}{\theta+\eta}
$$

Now, $\hat{q_{2}}=x^{-1} Q-\frac{\left(1-x^{-1}\right)\left(\alpha-\beta p_{1}\right)}{(\theta+\eta)}=q_{2}$ Thus, $\hat{q}_{2}=q_{2}$. Let us assume that $\hat{q}_{j-1}=q_{j-1}$. Then by induction,we can show that $\hat{q}_{j}=q_{j}$. Thus $q_{j}$ can be expressed as follows

$$
q_{j}=x^{-(j-1)} Q-\frac{\alpha\left(1-x^{-(j-1)}\right)}{(\theta+\eta)}+\frac{\left(1-x^{-1}\right) \sum_{k=2}^{j} x^{-(j-k)} \beta p_{k-1}}{\theta+\eta}
$$

Now we will develop the profit function which comprises of sales revenues, inventory holding cost, purchasing cost and pricing setting cost. From equations(2) and (5), we have

$$
\begin{aligned}
I_{j}(t)= & e^{-(\theta+\eta) t}\left[x^{-(j-1)} Q-\frac{\alpha\left(1-x^{-(j-1)}\right)}{(\theta+\eta)}+\frac{\left(1-x^{-1}\right) \sum_{k=2}^{j} x^{-(j-k)} \beta p_{k-1}}{\theta+\eta}\right] \\
& -\frac{\left(\alpha-\beta p_{j}\right)\left(1-\exp ^{-(\theta+\eta) t}\right)}{(\theta+\eta)}
\end{aligned}
$$




\section{Sales Revenues}

Let $\triangle q_{j}$ denote the sales amount during period $\mathrm{j}$. Then

$$
\begin{aligned}
\triangle q_{j}= & q_{j}-q_{j+1} \\
= & x^{-(j-1)} Q-x^{-j} Q-\frac{\alpha\left(1-x^{-(j-1)}\right)}{(\theta+\eta)}+\frac{\alpha\left(1-x^{-j}\right)}{(\theta+\eta)} \\
& \quad+\frac{\left(1-x^{-1}\right) \sum_{k=2}^{j} x^{-(j-k)} \beta p_{k-1}}{\theta+\eta}-\frac{\left(1-x^{-1}\right) \sum_{k=2}^{j+1} x^{-(j-k+1)} \beta p_{k-1}}{\theta+\eta} \\
= & x^{-j}(x-1) Q+\frac{\alpha\left(x^{-(j-1)}-x^{-j}\right)}{(\theta+\eta)} \\
& \quad+\frac{\beta\left(1-x^{-1}\right)}{\theta+\eta}\left(\sum_{k=2}^{j} x^{-(j-k)} p_{k-1}-\sum_{k=2}^{j+1} x^{-(j-k+1)} p_{k-1}\right) \\
= & x^{-j}(x-1) Q+\frac{\alpha\left(x^{-j}\right)(x-1)}{(\theta+\eta)}+\frac{\beta\left(1-x^{-1}\right)}{\theta+\eta}\left[\sum_{k=2}^{j} x^{-(j-k)} p_{k-1}\left(1-x^{-1}\right)-p_{j}\right] \\
= & \frac{\left(x^{-j}\right)(x-1)[Q(\theta+\eta)+\alpha]}{(\theta+\eta)}+\frac{\beta\left(1-x^{-1}\right)}{\theta+\eta}\left(\sum_{k=2}^{j}\left(1-x^{-1} x^{-(j-k)} p_{k-1}-\frac{\beta p_{j}\left(1-x^{-1}\right)}{\theta+\eta}\right)\right. \\
= & \frac{\left(x^{-j}\right)(x-1)[Q(\theta+\eta)+\alpha]}{(\theta+\eta)}-\frac{\beta p_{j}\left(1-x^{-1}\right)}{\theta+\eta}+\frac{\beta\left(1-x^{-1}\right)^{2}}{\theta+\eta}\left(\sum_{k=1}^{j-1}\left(x^{-(j-k-1)} p_{k}\right)\right.
\end{aligned}
$$

Let $\overline{R(n)}$ be the sales revenue when the firm divides the sales season into n-periods.

Then we have

$$
\begin{aligned}
\overline{R(n)}= & \sum_{j=1}^{n} \triangle q_{j} p_{j} \\
= & (x-1)\left(Q+\frac{\alpha}{(\theta+\eta)}\right) \sum_{j=1}^{n} x^{-j} p_{j}-\frac{\beta\left(1-x^{-1}\right) \sum_{j=1}^{n} p_{j}^{2}}{(\theta+\eta)} \\
& +\frac{\beta\left(1-x^{-1}\right)^{2}\left(\sum_{j=2}^{n} \sum_{k=1}^{j-1} x^{-(j-k-1)} p_{k} p_{j}\right)}{(\theta+\eta)}
\end{aligned}
$$

\section{Inventory Carrying Cost}

Let $\bar{H}_{j}(n)$ be the carrying cost of period $\mathrm{j}$ when the firm divides the sales season into $\mathrm{n}$ periods. Then

$$
\begin{aligned}
\bar{H}_{j}(n)= & \int_{t=0}^{T} I_{j}(t) h d t \\
= & {\left[x^{-(j-1)} Q-\frac{\alpha\left(1-x^{-(j-1)}\right)}{(\theta+\eta)}+\beta\left(1-x^{-1}\right) \sum_{k=2}^{j} x^{-(j-k)} p_{k-1}\right] \frac{\left(1-x^{-1}\right) h}{(\theta+\eta)} } \\
& -\frac{\left(\alpha-\beta p_{j}\right) h T}{(\theta+\eta)}+\frac{\left(\alpha-\beta p_{j}\right) h\left(1-x^{-1}\right)}{(\theta+\eta)^{2}}
\end{aligned}
$$


Thus

$$
\begin{aligned}
\bar{H}(n)= & \sum_{j=1}^{n} \bar{H}_{j}(n) \\
= & \frac{\left[\left(1-x^{-n}\right)(\alpha+(\theta+\eta) Q)+\beta\left(1-x^{-1}\right)^{2} \sum_{j=1}^{n} \sum_{k=2}^{j} x^{-(j-k)} p_{k-1}\right] h}{(\theta+\eta)^{2}} \\
& \quad-\frac{\beta\left(1-(\theta+\eta) T-x^{-1}\right) \sum_{j=1}^{n} p_{j} h}{(\theta+\eta)^{2}}-\frac{n T \alpha h}{(\theta+\eta)}
\end{aligned}
$$

where $\bar{H}(n)$ is the total carrying cost when the firm divides the sales season into n periods. Let $F(n, p, Q)$ be the total profit when the firm divides the sales season into n periods. Then, we have

$$
F(n, p, Q)=\bar{R}(n)-\bar{H}(n)-Q(n) c-n K .
$$

Note that the inventory level at the ending time of period $\mathrm{n}$ is zero. Thus, we have $I_{n}(T)=0$. Let $\mathrm{Q}(\mathrm{n})$ be the solution to the equation $I_{n}(T)=0$. Then, we obtain Then

$$
Q(n)=\frac{\alpha\left(x^{n}-1\right)-\beta\left(1-x^{-1}\right) \sum_{k=1}^{n} x^{k} p_{k}}{(\theta+\eta)}
$$

Substituting $Q=Q(n)$ into $\bar{R}(n)$ and $\bar{H}(n)$ and letting $F(n, p)$ be the result

$$
F(n, p)=R(n)-H(n)-Q(n) c-n K
$$

where

$$
\begin{aligned}
R(n)= & (x-1)\left(\frac{\alpha\left(x^{n}-1\right)-\beta\left(1-x^{-1}\right) \sum_{k=1}^{n} x^{k} p_{k}}{(\theta+\eta)}+\frac{\alpha}{(\theta+\eta)}\right) \sum_{j=1}^{n} x^{-j} p_{j} \\
& -\frac{\beta\left(1-x^{-1}\right) \sum_{j=1}^{n} p_{j}^{2}}{(\theta+\eta)}+\frac{\beta\left(1-x^{-1}\right)^{2}\left(\sum_{j=2}^{n} \sum_{k=1}^{j-1} x^{-(j-k-1)} p_{k} p_{j}\right)}{(\theta+\eta)} \\
H(n)= & {\left[\frac{\left(1-x^{-n}\right)\left(\alpha x^{n}-\beta\left(1-x^{-1}\right) \sum_{k=1}^{n} x^{k} p_{k}\right)}{(\theta+\eta)^{2}}\right] h } \\
& +\left[\frac{\beta\left(1-x^{-1}\right)^{2} \sum_{j=1}^{n} \sum_{k=2}^{j} x^{-(j-k)} p_{k-1}}{(\theta+\eta)^{2}}\right] h \\
& -\left[\frac{\beta\left(1-(\theta+\eta) T-x^{-1}\right) \sum_{j=1}^{n} p_{j}}{(\theta+\eta)^{2}}\right] h-\frac{n T \alpha h}{(\theta+\eta)}
\end{aligned}
$$

We discuss below the cases of no price change $(n=1)$, single price change $(n=2)$ and two price changes $(n=3)$.

Inventory Model Without Price Change

In this section, we assume that the firm sets its selling price at the start of the sales season and does not reset its selling price thereafter. Substituting $n=1$ into (13) and using (14) and (15), we have 


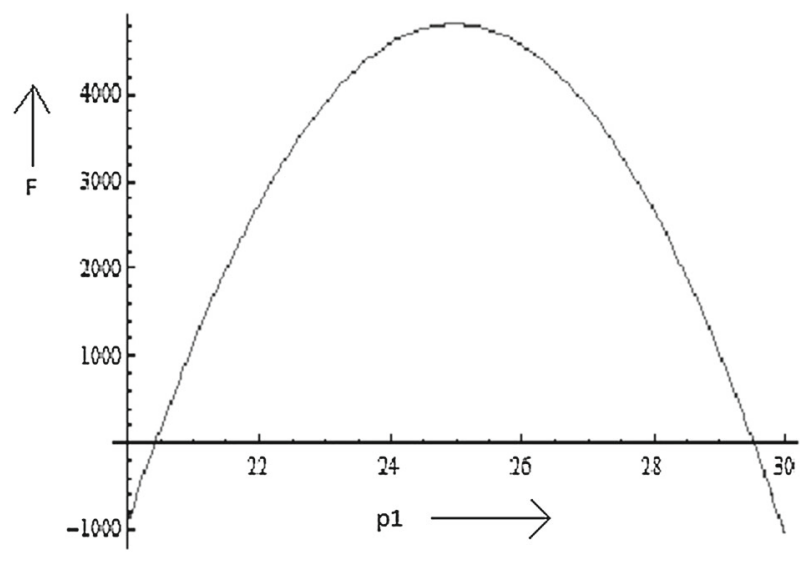

Graph for numerical 1

Fig. 1 Graphical representation of numerical example 1

$$
\begin{aligned}
F\left(1, p_{1}\right)=- & \frac{\beta(x-1) p_{1}^{2}}{(\theta+\eta)}+\frac{(x-1)\{\alpha(\theta+\eta)+\beta(c(\theta+\eta)+h)-\beta T h(\theta+\eta)\}}{(\theta+\eta)^{2}} p_{1} \\
& +\frac{\alpha T h}{(\theta+\eta)}-\frac{\alpha(x-1)\{c(\theta+\eta)+h\}}{(\theta+\eta)^{2}}-K
\end{aligned}
$$

Taking first and second order derivatives of $F\left(1, p_{1}\right)$ with respect to $p_{1}$, we get

$$
\frac{d F\left(1, p_{1}\right)}{d p_{1}}=\frac{-2 \beta(x-1) p_{1}}{(\theta+\eta)}+\frac{(x-1)(\alpha(\theta+\eta)+\beta(c(\theta+\eta)+h))}{(\theta+\eta)^{2}}-\frac{\beta T h}{(\theta+\eta)}
$$

and

$$
\frac{d^{2} F\left(1, p_{1}\right)}{d\left(p_{1}\right)^{2}}=\frac{-2(x-1) \beta}{(\theta+\eta)}<0
$$

Let $p_{j}(n)$ be the optimal selling price for period $\mathrm{j}$ when the firm divides the sales season into $\mathrm{n}$ periods. Then, since $\frac{d^{2} F\left(1, p_{1}\right)}{d\left(p_{1}\right)^{2}}<0$, objective function is concave in $p_{1}$. Accordingly, the optimal selling price is given by the solution to the first order condition. The necessary condition that $F\left(1, p_{1}\right)$ is maximum is given by $\frac{d F\left(1, p_{1}\right)}{d p_{1}}=0$. Then, we have

$$
p_{1}(1)=\frac{\alpha(\theta+\eta)+\beta(c(\theta+\eta)+h)}{2 \beta(\theta+\eta)}+\frac{T h}{2(1-x)}
$$

Substituting $p_{1}=p_{1}(1)$ in (12)

$$
Q(1)=\frac{\beta T h}{2(\theta+\eta)}+\frac{(x-1)\{\alpha(\theta+\eta)-\beta(c(\theta+\eta)+h)\}}{2(\theta+\eta)^{2}}
$$

Example 1 Suppose $L=100$ days, $h=\$ 0.002, \alpha=30, \beta=1.0, \eta=0.005, c=$ $\$ 20, K=\$ .80$ and $\theta=0.01$. Then, from (19) and (20) we have $p_{1}(1)=\$ 25.0379$ and $Q(1)=1151.76$, respectively. Substituting $p_{1}(1)$ into (16) we obtain that the optimal profit is $F\left(1, p_{1}(1)\right)=\$ 5635.07$. This result is is also shown graphically using the Fig. 1 . 
In the case of seasonal products like air conditioners, refrigerators, woollen clothes, seasonal vegetables, seasonal fruits etc, the price of the commodities varies depending on the demand and as well as the preservation cost. More precisely, woollen clothes are sold at a discounted rate during summer but with onset of winter they will cost more. Similarly, air conditioners and refrigerators will cost more in summer and less in winter. Also, off season vegetables and fruits costs more due to the preservation cost involved.

Inventory Model with a Single Price Change

Assume that the firm sets its selling price at the start of the sales season and resets its selling price at the time of $0.5 \mathrm{~L}$. Substituting $n=2$ into (13) we have

$$
\begin{aligned}
F\left(2, p_{1}, p_{2}\right)= & \frac{-\beta(x-1)\left(p_{1}^{2}+p_{2}^{2}\right)}{\theta+\eta}-\frac{\beta(x-1)^{2} p_{1} p_{2}}{\theta+\eta} \\
& +\frac{(x-1)(x \alpha(\theta+\eta)+\beta c(\theta+\eta)+\beta h) p_{1}}{(\theta+\eta)^{2}} \\
& +\frac{(x-1)(\alpha(\theta+\eta)+x \beta c(\theta+\eta)+x \beta h) p_{2}}{(\theta+\eta)^{2}}-\frac{\beta h T\left(p_{1}+p_{2}\right)}{(\theta+\eta)} \\
& +\frac{2 \alpha T h}{(\theta+\eta)}-\frac{\alpha\left(x^{2}-1\right)(c(\theta+\eta)+h)}{(\theta+\eta)^{2}}-2 K
\end{aligned}
$$

The Hessian matrix of $F\left(2, p_{1}, p_{2}\right)$ is given by

$$
H_{2}=\left(\begin{array}{ll}
\frac{-2 \beta(x-1)}{\theta+\eta} & \frac{-\beta(x-1)^{2}}{\theta+\eta} \\
\frac{-\beta(x-1)^{2}}{\theta+\eta} & \frac{-2 \beta(x-1)}{\theta+\eta}
\end{array}\right)
$$

Here $\frac{\partial^{2} F\left(2, p_{1}, p_{2}\right)}{\partial p_{1}^{2}}<0$ and $|H 2|=\frac{-\beta^{2}(x-1)^{2}(x+1)(x-3)}{(\theta+\eta)^{2}}>0$ for $x<3$.

Thus, $\mathrm{F}$ is concave function of selling prices when $x=e^{(\theta+\eta) T}=e^{(\theta+\eta) 0.5 L}<3$. Accordingly, for $x<3$, the optimal selling prices are given by the solutions to the first order conditions

$$
\begin{aligned}
\frac{\partial F\left(2, p_{1}, p_{2}\right)}{\partial p_{1}}= & \frac{-\beta(x-1)\left(2 p_{1}+(x-1) p_{2}\right)}{(\theta+\eta)}-\frac{\beta h T}{(\theta+\eta)} \\
& +\frac{(x-1)(x \alpha(\theta+\eta)+\beta c(\theta+\eta)+\beta h)}{(\theta+\eta)^{2}}=0
\end{aligned}
$$

and

$$
\begin{aligned}
\frac{\partial F\left(2, p_{1}, p_{2}\right)}{\partial p_{2}}= & \frac{-\beta(x-1)\left((x-1) p_{1}+2 p_{2}\right)}{(\theta+\eta)}-\frac{\beta h T}{(\theta+\eta)} \\
& +\frac{(x-1)(\alpha(\theta+\eta)+x \beta c(\theta+\eta)+x \beta h)}{(\theta+\eta)^{2}}=0
\end{aligned}
$$

Solving the above equation system gives

$$
\begin{array}{r}
p_{1}(2)=\frac{(x-2)(h+c(\theta+\eta))}{(\theta+\eta)(x-3)}-\frac{T h}{(x-1)(x+1)}-\frac{\alpha}{\beta(x-3)} \\
p_{2}(2)=\frac{\alpha(x-2)}{\beta(x-3)}-\frac{(h+c(\theta+\eta))}{(\theta+\eta)(x-3)}-\frac{T h}{(x-1)(x+1)}
\end{array}
$$




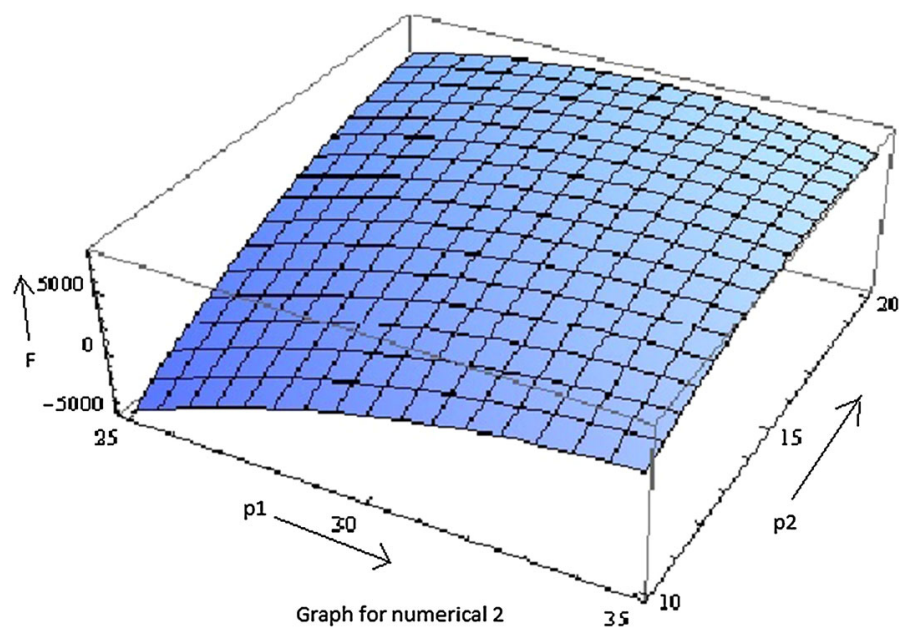

Fig. 2 Graphical representation of numerical example 2

Substituting $p_{1}=p_{1}(2)$ and $p_{2}=p_{2}(2)$ into (12), we have

$$
Q(2)=\frac{2(x-1)[\beta(h+c(\theta+\eta))-\alpha(\theta+\eta)]}{(x-3)(\theta+\eta)^{2}}+\frac{\beta T h}{(\theta+\eta)}
$$

Example 2 Suppose the parameters are the same as Example 1. It is noted that $x=$ $e^{(\theta+\eta) 0.5 L}=2.117<3$. Thus, the optimal selling prices are given by the solutions to the first order conditions. From (25) to (27) we have $p_{1}(2)=\$ 31.2786, p_{2}(2)=\$ 18.7973$ and $Q(2)=1670.85$, respectively. Substituting $p_{1}(2)$ and $p_{2}(2)$ into (21) we obtain that the optimal profit is $F\left(2, p_{1}(2), p_{2}(2)\right)=\$ 8115.95$. This result is shown graphically by Fig. 2 .

\section{Inventory Model with Two Price Changes}

In this section, we assume that the firm sets its selling price at the start of the sales season and resets its selling prices at the times of $\mathrm{L} / 3$ and $2 / 3 \mathrm{~L}$, respectively. Substituting $n=3$ into (13) we have

$$
\begin{aligned}
F\left(3, p_{1}, p_{2}, p_{3}\right)= & \frac{-\beta(x-1)\left(p_{1}^{2}+p_{2}^{2}+p_{3}^{2}\right)}{\theta+\eta}-\frac{\beta(x-1)^{2}\left(p_{1} p_{2}+p_{2} p_{3}+x p_{1} p_{3}\right)}{\theta+\eta} \\
& +\frac{\left[\alpha(\theta+\eta)\left(x^{3}-x^{2}\right)+x \beta\{h+c(\theta+\eta)\}-\beta\{h+T h(\theta+\eta)+c(\theta+\eta)\}\right] p_{1}}{(\theta+\eta)^{2}} \\
& +\frac{\left[\{\alpha(\theta+\eta)+\beta c(\theta+\eta)+\beta h\}\left(x^{2}-x\right)-\beta T h(\theta+\eta)\right] p_{2}}{(\theta+\eta)^{2}} \\
& +\frac{\left[\beta\{h+c(\theta+\eta)\}\left(x^{3}-x^{2}\right)+\alpha(\theta+\eta) x-\beta T h(\theta+\eta)-\alpha(\theta+\eta)\right] p_{3}}{(\theta+\eta)^{2}} \\
& -\frac{\alpha h(x-1)\left(x^{2}+x+1\right)}{(\theta+\eta)^{2}}+\frac{3 T h \alpha}{(\theta+\eta)}-\frac{\alpha\left(x^{3}-1\right) c}{(\theta+\eta)}-3 K
\end{aligned}
$$


The Hessian matrix of $F\left(3, p_{1}, p_{2}, p_{3}\right)$ is given by

$$
H_{3}=\left(\begin{array}{ccc}
\frac{-2 \beta(x-1)}{\theta+\eta} & \frac{-\beta(x-1)^{2}}{\theta+\eta} & \frac{-\beta x(x-1)^{2}}{\theta+\eta} \\
\frac{-\beta(x-1)^{2}}{\theta+\eta} & \frac{-2 \beta(x-1)}{\theta+\eta} & \frac{-\beta(x-1)^{2}}{\theta+\eta} \\
\frac{-\beta x(x-1)^{2}}{\theta+\eta} & \frac{-\beta(x-1)^{2}}{\theta+\eta} & \frac{-2 \beta(x-1)}{\theta+\eta}
\end{array}\right)
$$

Here, $\frac{\partial^{2} F\left(3, p_{1}, p_{2}, p_{3}\right)}{\partial p_{1}{ }^{2}}<0, \quad\left|H_{2}\right|=\frac{-\beta(x-1)^{2}(x+1)(x-3)}{(\theta+\eta)^{2}}>0$ for $x<3$ and $\left|H_{3}\right|=$ $\frac{2 \beta^{3}(x-1)^{3}(x+1)^{2}(x-2)}{(\theta+\eta)^{3}}<0$ for $x<2$.

Thus, $F\left(3, p_{1}, p_{2}, p_{3}\right)$ is concave function of selling prices when $x<2$. Accordingly, for $x=e^{(\theta+\eta) T}=e^{(\theta+\eta) L / 3}<2$, the optimal selling prices are given by the solution to the first order conditions. The first order conditions are:

$$
\begin{aligned}
\frac{\partial F\left(3, p_{1}, p_{2}, p_{3}\right)}{\partial p_{1}}= & \frac{-\beta(x-1)\left(2 p_{1}+(x-1)\left(p_{2}+x p_{3}\right)\right)}{(\theta+\eta)} \\
& +\frac{\left(\alpha(\theta+\eta)\left(x^{3}-x^{2}\right)+\beta x(c(\theta+\eta)+h)-\beta(h+(\theta+\eta) h T+c(\theta+\eta))\right.}{(\theta+\eta)^{2}}=0
\end{aligned}
$$

$$
\begin{array}{r}
\frac{\partial F\left(3, p_{1}, p_{2}, p_{3}\right)}{\partial p_{2}}=\frac{-\beta(x-1)\left((x-1)\left(p_{1}+p_{3}\right)+2 p_{2}\right)}{(\theta+\eta)}-\frac{\beta h T}{(\theta+\eta)} \\
+\frac{(\alpha(\theta+\eta)+\beta c(\theta+\eta)+\beta h)\left(x^{2}-x\right)}{(\theta+\eta)^{2}}=0
\end{array}
$$

$$
\begin{aligned}
\frac{\partial F\left(3, p_{1}, p_{2}, p_{3}\right)}{\partial p_{3}}= & \frac{-\beta(x-1)\left((x-1)\left(x p_{1}+p_{2}\right)+2 p_{3}\right)}{(\theta+\eta)}-\frac{\beta h T}{(\theta+\eta)} \\
& +\frac{\left(\beta(c(\theta+\eta)+h)\left(x^{3}-x^{2}\right)+\alpha(\theta+\eta) x-\alpha(\theta+\eta)\right.}{(\theta+\eta)^{2}}=0
\end{aligned}
$$

Solving the above system of equations

$$
\begin{aligned}
& p_{1}(3)=\frac{(2 x-3)(h+c(\theta+\eta))}{2(\theta+\eta)(x-2)}+\frac{(x-3) T h}{2(x-1)(x+1)}-\frac{\alpha}{2 \beta(x-2)} \\
& p_{2}(3)=\frac{\alpha(\theta+\eta)+\beta(c(\theta+\eta)+h)}{2 \beta(\theta+\eta)}-\frac{\left(x^{2}-3 x+4\right) T h}{2(x-1)(x+1)} \\
& p_{3}(3)=\frac{\alpha(2 x-3)}{2 \beta(x-2)}+\frac{(x-3) T h}{2(x-1)(x+1)}-\frac{h+c(\theta+\eta)}{2(\theta+\eta)(x-2)}
\end{aligned}
$$

Substituting $p_{1}=p_{1}(3), p_{2}=p_{2}(3)$ and $p_{3}=p_{3}(3)$ in (12), we have

$$
Q(3)=\frac{3(x-1)(\beta(h+c(\theta+\eta))-\alpha(\theta+\eta)}{2(x-2)(\theta+\eta)^{2}}+\frac{3 \beta T h}{2(\theta+\eta)}
$$

Example 3 Suppose the parameters are the same as Example 1. It is noted that $x=$ $e^{(\theta+\eta) 0.33 L}=1.6405<2$. Thus, the optimal selling prices are given by the solutions to the first order conditions. From (33) to (36) we have $p_{1}(3)=\$ 33.8295, p_{2}(3)=$ $\$ 25.0321, p_{3}(3)=\$ 16.2508$ and $Q(3)=1764.47$, respectively. Substituting $p_{1}(3), p_{2}(3)$, $p_{3}(3)$ and $\mathrm{Q}(3)$ into (28) we obtain that the optimal profit is $F\left(3, p_{1}(3), p_{2}(3), p_{3}(3)\right)=$ $\$ 8437.48$. 


\section{Sensitivity Analysis}

We refer to the data set used in the above example as the basic data set,We investigate the changes in the optimal decision values of $p_{1}(3), p_{2}(3), p_{3}(3), Q(3)$ and $F\left(3, p_{1}(3), p_{2}(3), p_{3}(3)\right)$ when only one parameter in the set $\omega$ where $[\omega: L=100$, $h=0.002, \alpha=30, \beta=1.0, \eta=0.005, c=20, K=80, \theta=0.01]$ changes and others remain unchanged. The computational results are described in Tables 1, 2, 3, 4, 5, 6, 7 and 8. In Example 3, the firm has two chances to adjust its selling prices. We refer the three selling prices as initial, secondary and final selling prices.

Table 1 shows that the initial selling price $p_{1}(3)$ decreases and secondary selling price $p_{2}(3)$ remains almost the same as L varies from +20 to $-20 \%$, and the final selling price $p_{3}(3)$ increases in L. This phenomenon could be explained as follows: First, we consider the situation in which the firm has a longer selling season as situation-A and the situation in which the firm has a shorter selling season as situation-B. Note that the firm would expect to obtain higher unit profit.Compared with situation-B, situation-A gives the firm longer time to achieve this goal. Thus, it can be conjectured that the firm in situation-A may set higher

Table 1 Sensitivity analysis for $\mathrm{L}$

Table 2 Sensitivity analysis for $\mathrm{h}$

Table 3 Sensitivity analysis for $\alpha$

Table 4 Sensitivity analysis for $\beta$

\begin{tabular}{lllllll}
\hline$i$ & $\%$ & $p_{1}(3)$ & $p_{2}(3)$ & $p_{3}(3)$ & $Q(3)$ & $F(3)$ \\
\hline 1 & +20 & 46.245 & 25.035 & 3.85 & 4247.76 & 20754.9 \\
2 & +10 & 37.968 & 25.034 & 12.119 & 2592.19 & 12584.1 \\
3 & -10 & 31.348 & 25.030 & 18.726 & 1268.19 & 6045.91 \\
4 & -20 & 29.696 & 25.028 & 20.371 & 937.71 & 4412.27 \\
\hline
\end{tabular}

\begin{tabular}{lllllll}
\hline$i$ & $\%$ & $p_{1}(3)$ & $p_{2}(3)$ & $p_{3}(3)$ & $Q(3)$ & $F(3)$ \\
\hline 1 & +20 & 33.814 & 25.039 & 16.283 & 1761.04 & 8463.57 \\
2 & +10 & 33.822 & 25.035 & 16.267 & 1762.76 & 8480.52 \\
3 & -10 & 33.837 & 25.029 & 16.235 & 1766.19 & 8514.47 \\
4 & -20 & 33.845 & 25.026 & 16.219 & 1767.9 & 8531.47 \\
\hline
\end{tabular}

\begin{tabular}{llllllc}
\hline$i$ & $\%$ & $p_{1}(3)$ & $p_{2}(3)$ & $p_{3}(3)$ & $Q(3)$ & $F(3)$ \\
\hline 1 & +20 & 42.174 & 28.032 & 13.906 & 2833.45 & 22291.2 \\
2 & +10 & 38.002 & 26.532 & 15.078 & 2298.96 & 14592.6 \\
3 & -10 & 29.657 & 23.532 & 17.423 & 1229.98 & 4005.8 \\
4 & -20 & 25.485 & 25.032 & 18.596 & 695.496 & 1117.58 \\
\hline
\end{tabular}

\begin{tabular}{llllllc}
\hline$i$ & $\%$ & $p_{1}(3)$ & $p_{2}(3)$ & $p_{3}(3)$ & $Q(3)$ & $F(3)$ \\
\hline 1 & +20 & 26.875 & 22.532 & 18.205 & 1048.39 & 2330.59 \\
2 & +10 & 30.036 & 23.669 & 17.317 & 1406.43 & 4806.66 \\
3 & -10 & 38.466 & 26.699 & 14.948 & 2122.51 & 13808.0 \\
4 & -20 & 44.261 & 28.782 & 13.320 & 2480.55 & 21345.5 \\
\hline
\end{tabular}


Table 5 Sensitivity analysis for $\eta$

Table 6 Sensitivity analysis for $c$

Table 7 Sensitivity analysis for $k$

Table 8 Sensitivity analysis for $\theta$

\begin{tabular}{llllllc}
\hline$i$ & $\%$ & $p_{1}(3)$ & $p_{2}(3)$ & $p_{3}(3)$ & $Q(3)$ & $F(3)$ \\
\hline 1 & +20 & 36.319 & 25.031 & 13.76 & 2121.12 & 10263.7 \\
2 & +10 & 34.961 & 25.032 & 15.119 & 1926.60 & 9300.38 \\
3 & -10 & 32.873 & 25.033 & 17.208 & 1627.28 & 7818.03 \\
4 & -20 & 32.053 & 25.033 & 18.029 & 1509.67 & 7235.58 \\
\hline
\end{tabular}

\begin{tabular}{lllllll}
\hline$i$ & $\%$ & $p_{1}(3)$ & $p_{2}(3)$ & $p_{3}(3)$ & $Q(3)$ & $F(3)$ \\
\hline 1 & +20 & 32.266 & 27.032 & 21.814 & 1051.82 & 2864.9 \\
2 & +10 & 33.048 & 26.032 & 19.032 & 1408.15 & 5324.86 \\
3 & -10 & 34.611 & 24.032 & 13.469 & 2120.8 & 12382.8 \\
4 & -20 & 35.393 & 23.032 & 10.688 & 2477.12 & 16980.7 \\
\hline
\end{tabular}

\begin{tabular}{lllllll}
\hline$i$ & $\%$ & $p_{1}(3)$ & $p_{2}(3)$ & $p_{3}(3)$ & $Q(3)$ & $F(3)$ \\
\hline 1 & +20 & 33.83 & 25.0321 & 16.251 & 1764.47 & 8449.48 \\
2 & +10 & 33.83 & 25.032 & 16.251 & 1764.47 & 8473.48 \\
3 & -10 & 33.83 & 25.032 & 16.251 & 1764.47 & 8521.48 \\
4 & -20 & 33.83 & 25.032 & 16.251 & 1764.47 & 8545.48 \\
\hline
\end{tabular}

\begin{tabular}{lllllll}
\hline$i$ & $\%$ & $p_{1}(3)$ & $p_{2}(3)$ & $p_{3}(3)$ & $Q(3)$ & $F(3)$ \\
\hline 1 & +20 & 40.056 & 25.03 & 10.022 & 2655.89 & 12912.1 \\
2 & +10 & 36.3192 & 25.03 & 13.76 & 2121.12 & 10263.7 \\
3 & -10 & 32.053 & 25.03 & 18.029 & 1509.67 & 7235.58 \\
4 & -20 & 30.722 & 25.03 & 19.361 & 1318.53 & 6289 \\
\hline
\end{tabular}

initial and secondary selling prices to achieve higher unit profit. It can also be conjectured that the remaining inventory in situation-A may be larger than that in situation-B when the final selling price is to be set. To sell out its inventory, the firm in situation-A would set a lower final selling price to sell its items.According to this reason, the initial selling price $p_{1}(3)$ and secondary selling price $p_{2}(3)$ increase in the length of $\mathrm{L}$, and the final selling price $p_{3}(3)$ decreases in L.

Table 2 shows that the initial selling price $p_{1}(3)$ decreases when the value of $\mathrm{h}$ increases from -20 to $+20 \%$, and the secondary selling price $p_{2}(3)$ and the final selling price $p_{3}(3)$ increase when the value of $\mathrm{h}$ increases from -20 to $+20 \%$. This phenomenon could be explained as follows: First, we consider the situation in which the firm has a higher inventory carrying cost as situation-C and the situation in which the firm has a lower inventory carrying cost as situation-D. Note that the firm would aim to reduce its inventory holding cost, as spending more on holding cost leads to expenditure of the firm.Hence it lessens the profit.The inventory holding cost has a tight relationship with inventory level, inventory holding time and unit holding cost.Compared with situation-D, situation-C gives the firm more incentives to reduce its inventory when the time to sell its items is still long. It can be conjectured that the 
firm in situation-C may set a lower initial selling price to reduce its inventory. It can also be conjectured that the remaining inventory in situation- $\mathrm{C}$ may be less than that in situation-D when the secondary selling price is to be set. To obtain a higher unit profit, the firm then would set higher secondary and final selling prices to sell its items. According to this reason, the initial selling price $p_{1}(3)$ decreases in $\mathrm{h}$, and the secondary selling price $p_{2}(3)$ and the final selling price $p_{3}(3)$ increase in $\mathrm{h}$.

Table 3 shows that the initial selling price $p_{1}(3)$, secondary selling price $p_{2}(3)$ increase as $\alpha$ increases from -20 to $+20 \%$ and the final selling price $p_{3}(3)$ decreases in the value of $\alpha$. This phenomenon could be explained as follows. First, increasing the value of $\alpha$ moves the demand curve up. Thus, compared with an inventory system with a smaller value of $\alpha$, the firm in situation- $\mathrm{C}$ with a higher value of $\alpha$ may set higher selling prices to improve its unit profit. According to this reason, the initial selling price $p_{1}(3)$, the secondary selling price $p_{2}(3)$ increase and the final selling price $p_{3}(3)$ decreases in $\alpha$.

Table 4 shows that the initial selling price $p_{1}(3)$ and the secondary selling price $p_{2}(3)$ decreases when the value of $\beta$ increases from -20 to $+20 \%$, and the final selling price $p_{3}(3)$ increase when the value of $\beta$ increases from $-20 \%$ to $+20 \%$. This phenomenon could be explained as follows:higher value of $\beta$ has a reducing effect on demand.Hence to make some profit the initial price should be more so that the unit profit per sale is more.

Table 5 shows that the initial selling price $p_{1}(3)$ increases in the value of $\eta$, and the secondary selling price $p_{2}$ (3) and the final selling price $p_{3}(3)$ decrease in the value of $\eta$. This phenomenon could be explained as follows. First, we consider the situation in which the firm has a higher value of $\eta$ as situation-E and the situation in which the firm has a lower value of $\eta$ as situation-F. Note that the term of $\eta \mathrm{I}(\mathrm{t})$ has positive impact on demand. Initially, the inventory level for an inventory system is high. Under the same selling price, the demand rate in situation-E is higher than that in situation-F. Thus, it can be conjectured that the firm in situation-E may have more incentives to set a higher selling price to obtain higher unit profit. Once the secondary selling price is set, the firm in situation-E may have more stocks on hand. To reduce its inventory, it can be conjectured that the firm would set a lower secondary and final selling prices to reduce its inventory in situation-E. According to this reason, the initial selling price $p_{1}(3)$ increases in $\eta$, and the secondary selling price $p_{2}(3)$ and the final selling price $p_{3}(3)$ decrease in $\eta$.

Table 6 shows that the initial selling price $p_{1}(3)$ decreases whereas secondary selling price $p_{2}(3)$ and the final selling price $p_{3}(3)$ increase as the value of c varies from -20 to $+20 \%$. This phenomenon could be explained as follows: First, note that increasing the value of $\mathrm{c}$ reduces the unit profit. Thus, compared with an inventory system with a smaller value of $\mathrm{c}$, the firm with higher value of $\mathrm{c}$ may set higher selling prices to cover its unit cost and improve its unit profit. According to this reason, the initial selling price $p_{1}(3)$, the secondary selling price $p_{2}(3)$ and the final selling price $p_{3}(3)$ increases in $\alpha$.

From Tables 1, 2, 3, 4, 5 and 6 , we also see that the ordering quantity and the profit increase with $\mathrm{L}, \alpha$ and $\eta$ and decreases in $\mathrm{h}, \beta$ and $\mathrm{c}$. It could be explained as follows: As $\mathrm{L}$ increases, the firm has more time to sell its items. Thus, the firm orders more and gets more profit. Since the parameters of $\alpha$ and $\eta$ have positive effect on demand, the ordering quantity and the profit increase as $\alpha$ and $\eta$ increase. Since the parameters of $\mathrm{h}, \beta$ and $\mathrm{c}$ have negative effect on demand, the ordering quantity and the profit decrease when $\mathrm{h}$ and $\mathrm{c}$ increase.

Table 7 shows that the variation in price setting cost has no change in the values of initial selling price $p_{1}(3)$, the secondary selling price $p_{2}(3)$ and the final selling price $p_{3}(3)$ as order quantity.Although the profit reduces as price setting cost adds only to the expenditure of the firm which gets subtracted from the total profit. 
Table 8 shows that as $\theta$ varies from -20 to $+20 \%, p_{1}(3)$ increases whereas $p_{2}(3)$ remains same and $p_{3}(3)$ decreases. This phenomena can be explained as follows:as the deterioration rate increases ,loss due to deterioration also increases. Hence to make up with the loss higher price is set every time the deterioration rate increases. After certain period of time in the finite time zone, when the percentage of deteriorated items increase, in order to clear all stock the final selling price $p_{3}(3)$ is decreased.

Thus, the characteristics of the sensitivity analysis are summarized as follows:

(1) $p_{1}(3)$ increases with $\mathrm{L}, \alpha, \theta$ and $\eta$ while it decreases with $\mathrm{h}, \beta$ and c;

(2) $p_{2}$ (3) increases with $\mathrm{L}, \mathrm{h}, \alpha$ and $\mathrm{c}$ while it decreases with $\eta$ and $\beta$;

(3) $p_{3}(3)$ increases with $\mathrm{h}, \beta$ and $\mathrm{c}$ while it decreases with $\mathrm{L}, \alpha, \theta$ and $\eta$;

(4) $\mathrm{Q}(3)$ increases with $\mathrm{L}, \alpha$ and $\eta$ while it decreases with $\mathrm{h}, \beta, \theta$ and $\mathrm{c}$; and

(5) $F\left(3, p_{1}(3), p_{2}(3), p_{3}(3)\right)$ increases with $\mathrm{L}, \alpha, \beta, \theta$ and $\eta$ while it decreases with $\mathrm{h}, \mathrm{k}, \beta$ and $\mathrm{c}$.

Sensitivity analysis of numerical 3 .

\section{Conclusion}

In the proposed model, we have studied the pricing and ordering problem for an inventory system which is subjected to deterioration.Many inventory models have considered this problem. However these models have rarely considered cases in which sales price can be adjusted during the selling period and the number of price changes can be controlled.Also, another important aspect of this paper is that the items taken into account are subjected to deterioration.This fact adds to the loss areas of the firm. Thus the profit is reduced. In between price setting is allowed, which can be highered or lowered depending on whether to keep the stock or clear it respectively, keeping in mind "maximization of profit".

In the present work we do not consider deterioration to be time dependent. It seems necessary to reformulate our model by taking time-dependent deterioration. In addition,extension of the proposed model to unequal time price changes and other applications will be a focus of our future work.

\section{References}

1. Baker, R.C., Urban, T.L.: A deterministic inventory system with an inventory-level dependent demand rate. J. Oper. Res. Soc. 39, 823-831 (1988)

2. Bhunia, A.K., Maiti, M.: Deterministic inventory model for deteriorating items with finite rate of replenishment dependent on inventory level. Comput. Oper. Res. 25, 907-1006 (1998)

3. Chung, K.J., Chu, P., Lan, S.P.: A note on EOQ models for deteriorating items under stock dependent selling rate, European. J. Oper. Res. Soc. 124, 550-559 (2000)

4. Datta, T.K., Pal, A.K.: A note on an inventory model with inventory-level-dependent demand rate. J. Oper. Res. Soc. 41(10), 971-975 (1990)

5. Datta, T.K., Pal, A.K.: Effects of inflation and time-value of money on an inventory model with linear time dependent demand rate and shortages. Eur. J. Oper. Res. 52, 1-8 (1991)

6. Dye, C.-Y., Ouyang, L.-Y.: An EOQ model for perishable items under stock-dependent selling rate and time-dependent partial backlogging. Eur. J. Oper. Res. 163, 776-783 (2005)

7. Gupta, R., Vrat, P.: Inventory model for stock-dependent consumption rate. Opesearch 23, 19-24 (1986)

8. Levin, R.I., McLaughlin, C.P., Lamone, R.P., Kottas, J.F.: Productions/ Operations Management: Contemporary Policy for Managing Operating Systems. McGraw-Hill, New Work (1972)

9. Lin, K.Y.: Dynamic pricing with real-time demand learning. Eur. J. Oper. Res. 174(1), 522-538 (2006)

10. Mandal, M., Maiti, M.: Inventory of damagable items with variable replenishment rate, stock-dependent demand and some units in hand. Appl. Math. Model. 23, 799-807 (1999) 
11. Mishra, V.K.: An inventory model of instantaneous deteriorating items with controllable deterioration rate for time dependent demand and holding cost. J. Ind. Eng. Manag. 6, 495-506 (2013)

12. Mondal, B.N., Phaujdar, S.: A note on an inventory model with stock-dependent consumption rate. Opsearch 26, 43-46 (1989a)

13. Mondal, B.N., Phaujdar, S.: A inventory model for deteriorating items and stock-dependent consumption rate. J. Oper. Res. Soc. 40, 483-488 (1989b)

14. Pal, S., Goswami, A., Choudhuri, K.S.: A deterministic inventory model for deteriorating items with stock-dependent demand rate. Int. J. Prod. Econ. 32, 291-299 (1993)

15. Ray, J., Chaudhri, K.S.: An EOQ model with stock-dependent demand, shortages, inflation and timediscounting. Int. J. Prod. Econ. 53, 171-180 (1997)

16. Roy, T., Chaudhuri, K.S.: Deterministic inventory model for deteriorating items with stock-dependent demand, shortage, inflation and time discounting. Nonlinear Phenom. Complex Syst. 9(1), 43-52 (2006)

17. Sana, S.S.: An EOQ model for salesmen's initiatives, stock and price sensitive demand of similar products: a dynamical system. J. Appl. Math. Comput. 218(7), 3277-3288 (2011)

18. Sarkar, B.: An EOQ model with delay in payments and time varying deterioration rate. Math. Comput. Modell. 55, 367-377 (2012)

19. Sarkar, B.: An EOQ model with delay in payments and stock dependent demand in the presence of imperfect production. Appl. Math. Comput. 218, 8295-8308 (2012)

20. Sarkar, B., Moon, I.: An EPQ model with inflation in an imperfect production system. Appl. Math. Comput. 217, 6159-6167 (2011)

21. Sarkar, B.: A production-inventory model with probabilistic deterioration in two-echelon supply chain management. Appl. Math. Model. 37(5), 3138-3151 (2013)

22. Setta, B.K., Sarkar, B., Goswami, A.: A two-warehouse inventory model with increasing demand and time varying deterioration. Scientia Iranica 19(6), 1969-1977 (2012)

23. Silver, E.A., Peterson, R.: Decision Systems for Inventory Management and Production planning, 2nd edn. Wiley, New York (1985)

24. Urban, T.L., Baker, R.C.: Optimal ordering and pricing policies in a single-period environment with multivariate demand and markdowns. Eur. J. Oper. Res. 103, 573-583 (1997)

25. Urban, T.L.: An inventory model with an inventory-level-dependendent demand rate and relaxed terminal conditions. J. Oper. Res. Soc. 43(7), 721-724 (1992)

26. Urban, T.L.: Inventory models with inventory-level-dependent demand: a comprehensive review and unifying theory. Eur. J. Oper. Res. 162, 792804 (2005)

27. Urban, T.L.: A periodic-review model with serially-correlated, inventory-level-dependent demand. Int. J. Prod. Econ. 95, 287295 (2005) 